\title{
Community mental health teams in low- and middle-income countries
}

\author{
R. Thara ${ }^{1 *}$, S. John ${ }^{1}$ and S. Chatterjee ${ }^{2}$ \\ ${ }^{1}$ Schizophrenia Research Foundation (SCARF), Chennai, India \\ 2 Sangath, Goa, India
}

\begin{abstract}
The dearth of trained mental health professionals and the huge gap in providing accessible services in many low- and middle-income countries have led to the identification of alternate providers of care in these countries. Community mental health teams seem to fill this lacuna in some of these places. This editorial addresses issues of the need for such teams, their composition, responsibilities and limitations. With adequate training, these teams are able to carry out a broad array of tasks such a case identification, referrals, elementary counselling, family support and psychosocial interventions. While these teams are generally found to be enthusiastic, they require periodic monitoring and support with which they can well be a critical element of the mental health care team.
\end{abstract}

First published online 11 February 2014

Key words: Care providers, community teams, LAMIC, mental health.

The community mental health movement was a natural sequel to the process of deinstitutionalisation in the West in the 1960s and led to the development of specific services and facilities such as halfway houses and hostels. Catchment area-based multidisciplinary teams were created to attend to patients discharged into the community leading to the creation of the multidisciplinary case management and assertive community treatment (ACT) team concepts among others.

However the process was quite different in the lowand middle-income countries (LAMICs) of Asia, Africa and South America. In India, for example, people with mental disorders have been traditionally contained within the community with families being the main caregivers. The larger community and traditional healers have also played a role (Isaac, 2012). Even today, this is ubiquitous in the rural areas of many LAMIC facilitated by the greater tolerance for illness behaviour, lowered expectations and religion-based coping as well as healing strategies by the family and community (Isaac, 2012).

On the flip side, however, these countries have to contend with the lack of resources both in terms of manpower and budgetary resources resulting in inadequate number of psychiatric beds or staff to manage patients even in psychiatric hospitals. In LAMICs, there is on average one psychiatrist per 1.7 million inhabitants and one psychiatric inpatient bed per 42

* Address for correspondence: Dr R. Thara, Schizophrenia Research Foundation, SCARF Mental Health Centre, R/7A, North Main Road, Anna Nagar West Extension, Chennai 600101, India.

(Email: thara@scarfindia.org)
000 inhabitants (Jacob et al. 2007). Non-availability of formal $\mathrm{MH}$ services leads to utilisation of traditional or faith-based treatment methods. Even today, community mental health services are not a well-organised component of health systems in many LAMIC.

In the late-1970s, the National Institute of Mental Health and Neuro Sciences (NIMHANS) in India piloted a model of community care in Bellary which attempted to integrate basic mental health care within the general health care services. This model looked at decentralised training, involvement of the primary health care physician and utilising the services of lay health workers apart from making psychotropic medication available at the local health centre. This 'Bellary model' was later taken up for implementation of the District Mental Health Programme (DMHP), an essential arm of the National MHP.

The recommended DMHP team consists of a psychiatrist or a trained medical officer, a psychologist, social worker, a community nurse and a record keeper. Apart from this five-member team, two additional members (community nurse and an assistant) are allocated for coordination and implementation of activities in the district (Kaur \& Sinha, 2012).

The NMHP in India has not been a uniform success story. While most experts found that the programme failed to deliver on its promises due to a variety of reasons, others have pointed out that the programme has ensured wider availability of psychiatric medication (Jacob, 2010).

Several innovative strategies such as community mental health camps and extension clinics have 
subsequently been tried with varying degrees of success with regards to sustainability and transference of care. The primary reason for the non-sustainability of several of these efforts was the fact that they were psychiatrist-driven models (Patel, 2011).

In the absence of sufficient number of trained mental health professionals especially psychiatrists in the developing countries, scaling up of mental health services requires a mechanism to be put in place. The process of facilitating and sustaining the process of task shifting is critical. Locally and traditionally available resources including those existing for delivery of nonmedical services have been used for delivery of mental health services.

Keeping this in mind several programmes have been started in India and other developing countries such as Indonesia, Vietnam, Sri Lanka, etc. where lay volunteers from within the catchment area of the designated service are recruited and trained in identification of persons with mental disorders, making appropriate referrals, ensuring regular follow-up, conducting psycho-education sessions, creating awareness and facilitating rehabilitation. Their training was conceived as an ongoing process with the majority happening on the job apart from the initial few introductory sessions.

One of the largest deployments of lay workers to deliver psycho-social intervention happened following the devastating tsunami of 2004 that affected several nations. The World Health Organization (WHO) initiated and coordinated the relief efforts with the respective national and local governments and sought to deliver mental health care through those providing other relief measures. The effort was largely successful and paved the way in many of the affected nations to look at using lay workers to deliver mental health care.

The Schizophrenia Research Foundation (SCARF), a non-governmental, not for profit organisation in Chennai, India has used Community Level Workers (CLWs) in the delivery of several of its community outreach programmes in rural Tamil Nadu including during the post-tsunami interventions in the districts of Nagapattinam and Cuddlore. They were also extensively used in another semi-rural outreach programme at Thiruporur wherein they were trained to identify, refer, follow-up and rehabilitate patients with mental disorders. These activities were carried out through home visits and organisation of group sessions. Their other responsibilities included creating awareness through folk music, street plays, skits and other activities in keeping with the local culture and sensibilities. CLWs also conducted psycho-education programmes for family members and were responsible for nurturing linkages with other local community organisations and resources necessary for the global improvement of patients.
There have been several instances when the family had given up hopes of the patient improving and shackled them in chains due to community pressure. The CLWs through a process of constant engagement and regular dialogue with the family and community were able to convince them to free the patient from the chains and initiate psychiatric treatment. Several of these individuals have shown remarkable improvement after treatment and have become poster cases for the programme. Keeping in mind that 'outsiders' are generally viewed with suspicion it would have been an impossible task for anyone else apart from the CLWs (who are recruited from the local community) to convince the families to initiate treatment for their relative.

It has also been SCARF's experience that CLWs can also be successfully used to deliver technologyenabled services such as telepsychiatry following adequate training. The SCARF Telepsychiatry Programme (STEP) in Pudukottai relied upon CLWs to screen patients, collect basic history, conduct the teleclinic by linking it with the hub at Chennai, dispense medication and ensure follow-up.

SCARF has preferred to use young women with high school level of education as CLWs in several of its programmes. This has been guided by the fact that women are seen as less threatening and can more easily gain entry into rural households especially if the ill individual is also a woman.

The team of CLWs is managed by a qualified social worker who monitors their activities and provides professional supervision. The CLWs in turn work with volunteers and members of self-help group (SHG) clusters involved in micro-financing of livelihood activities that can facilitate the rehabilitation of the ill individuals within the community. As such the community mental health team comprises a flexible group comprising a mental health professional (not necessarily a psychiatrist), trained lay workers, volunteers and pre-existing SHGs.

Other example of successful involvement of CLWs working as a team with a Psychiatrist has been reported from a rural and socially disadvantaged part of India. A local non-governmental organisation (NGO) called 'Ashagram', based in Barwani district of the Indian state of Madhya Pradesh, developed and evaluated an innovative community based rehabilitation (CBR) model of service delivery for people with psychoses (Chatterjee et al. 2003, 2009). In this CBR programme, lay or non specialist CLWs, drawn from the local area, were trained and supervised to deliver interventions such as psychoeducation, ensuring timely follow-up, rehabilitation, adherence management, improving access to employment opportunities, networking with community agencies for 
social and social inclusion. In addition, CLWs helped develop transactional, micro-credit-based SHGs in each of the catchment area villages for people with severe mental disorders and the general community. CLWs were successfully trained to identify and refer persons for specialist assessments, deliver accessible, need based interventions in collaboration with the specialists, help develop mechanisms for initiating collective action through organising SHGs and to engage with the community to enhance social inclusion and developmental opportunities. In addition, the Ashagram experience provided observational evidence that demonstrated the superiority of the CBR services as compared with routine outpatient care for the first time from a LAMIC setting.

Several programmes have also attempted to involve traditional and faith healers as partners in the delivery of mental health care with some limited success. For example, SCARF in one of its programmes, worked with religious healing centres; while the religious treatment continued as planned, volunteers at the place of worship helped persuade patients and families to add psychotropic medication to their current religious treatment (Thara, 2002). The Sowmanasya Hospital, a private enterprise in Tiruchy, Tamil Nadu also has worked with traditional and religious centres and, with the help of the temple priest, established a psychiatry outreach within the temple (Stanley \& Shwetha, 2006).

Sri Lanka has used a different strategy to tackle the manpower shortage in mental health service delivery. They have created a cadre called the Medical Officers of Mental Health (MOMH) who are newly qualified physicians who after receiving 6 months of training in mental health are posted at rural hospital (Mendis, 2012). They now work within the community and make home visits, school visits and visit institutions dedicated to children and the elderly. Now a new group of physicians are being trained under a recently created 1 year diploma programme in psychiatry.

Apart from this the Sri Lankan Ministry of Health in 2010 organised training in mental health for two nurses from each district to create a cadre of community psychiatric nurses (CPNs) who will work in the community with psychiatrists and MOMHs. However, due to the need for supervision most of these nurses are placed where psychiatrists are available rather than with MOMHs.

Another group of workers that was instituted were the Community Support Officers (CSOs) who similar to the CLWs are lay volunteers who have been trained in mental health. The CSOs were created in the aftermath of the tsunami to provide psycho-social interventions.

Apart from this there are psychiatric social workers who are present in some of the psychiatrist lead teams but due to their acute shortage most community teams do not have a trained social worker. There have also been several calls to use the public health midwifes (PHM), community nurses called Public Health Sister (PHS) and health inspectors in the delivery of mental health care in the community.

The situation in Pakistan is similar to that faced by its neighbours - an acute shortage of mental health professionals. Pakistan has followed a strategy similar to Sri Lanka and has focused on training general physicians in psychiatry. Since 2005, a one year certificate course in psychiatry is being offered in collaboration with the World Psychiatry Association (WPA). Apart from this Continuous Medical Education (CME) programmes are being conducted in 35 cities to up skill primary care physicians in psychiatry (Chaudhry et al. 2012).

In addition, for the past decade and a half-regular outreach and awareness programmes have been conducted under the 'Reaching the unreached' platform where psychiatry camps have been conducted and free medicines dispensed. This was done in alliance with psychiatrists, primary care physicians, health care workers, clinical psychologists, students, social workers and the pharmaceutical industry.

There are several NGOs in Pakistan involved in community-based care and several different models are being followed including the Fountain House Project modelled on the New York Fountain House Model.

As is often the case, the shortage of mental health professionals, especially in rural areas, has resulted in large sections of the population turning to traditional and religious healers for relief and treatment. While the medical establishment understands the usefulness of roping in them as partners to reach out to the population, there is a lot of hesitation and reluctance to put this approach into practice and as such this remains a vast untapped resource.

Smaller nations such as Singapore which have made rapid economic strides and have transcended from low-income to high-income bracket have steadily been able to build up its community mental health programmes and teams modelled on the Community Mental Health Teams (CMHTs) of the West. These include the ACT team composed of multidisciplinary professionals that include a consultant psychiatrist, medical officer, CPNs, medical social worker and an occupational therapist. They provide treatment monitoring, rehabilitation and other support services through home visits. The Institute of Mental Health (IMH) of Singapore also runs a helpline which families can access to obtain immediate advice and a Mobile Crisis Team (MCT), which consisted of two psychiatric nurses and a medical officer or social worker when needed. The Singapore Association for Mental Health (SAMH) established in 1969 now runs services that 
include group home, social clubs day activity and rehab centres (Sengupta et al. 2012). This model will not be replicable in other nations which are much larger and have greater populations to serve.

We strongly believe that resource poor countries should look to evolve its own community psychiatry model with its scarce trained manpower resources deployed for optimum results. This dictates the need for the creation of indigenous community mental health teams wherein a process of task shifting is accomplished by utilising and partnering locally available resources such as lay health workers and traditional healers, apart from incorporating home-grown micro-financing schemes for entrepreneurs for rehabilitation purposes rather than replicate the models developed in the high-resource countries.

\section{Financial Support}

This research received no specific grant from any funding agency, commercial or not-for-profit sectors.

\section{Conflict of Interest}

None.

\section{Ethical Standards}

The authors assert that all procedures contributing to this work comply with the ethical standards of the relevant national and institutional committees on human experimentation and with the Helsinki Declaration of 1975, as revised in 2008.

\section{References}

Chatterjee S, Patel V, Chatterjee A, Weiss H (2003).

Evaluation of a community-based rehabilitation model for chronic schizophrenia in rural India. British Journal of Psychiatry 182, 57-62.

Chatterjee S, Pillai A, Jain S, Cohen A, Patel V (2009). Outcomes of people with psychotic disorders in a community-based rehabilitation programme in rural India. British Journal of Psychiatry 195, 433-439.

Chaudhry HR, Khan RM, Shabbir A (2012). Community psychiatry in Pakistan. In Community Mental Health in India (ed. BS Chavan, N Gupta, P Arun, A Sidana and S Jadhav), pp. 485-490. Jaypee: New Delhi.

Isaac M (2012). Introduction. In Community Mental Health in India (ed. BS Chavan, N Gupta, P Arun, A Sidana and S Jadhav), pp. xxxv-xli. Jaypee: New Delhi.

Jacob KS (2010). Repackaging mental health programs. In Opinion, Hindu November 4.

Jacob KS, Sharan P, Mirza I, Garrido-Cumbrera M, Seedat S, Mari JJ, Sreenivas V, Saxena S (2007). Mental health systems in countries: where are we now? Lancet 370, 10611077.

Kaur J, Sinha SK (2012). Community mental health initiatives by the government: past, present and future. In Community Mental Health in India (ed. BS Chavan, N Gupta, P Arun, A Sidana and S Jadhav), pp. 383-392. Jaypee: New Delhi.

Mendis J (2012). Community psychiatry in Sri Lanka. In Community Mental Health in India (ed. BS Chavan, N Gupta, P Arun, A Sidana and S Jadhav), pp. 478-484. Jaypee: New Delhi.

Patel V (2011). The great push for mental health: why it matters for India. Indian Journal of Medical Research 134, 26-35.

Sengupta S, Leong Jern-Yi J, Cheng L (2012). Community psychiatry in Singapore. In Community Mental Health in India (ed. BS Chavan, N Gupta, P Arun, A Sidana and S Jadhav), pp. 460-469. Jaypee: New Delhi.

Stanley S, Shwetha S (2006). Integrated psychosocial intervention in Schizophrenia: implications for patients and caregivers. International Journal of Psychosocial Rehabilitation 10, 113-128.

Thara R (2002). Community mental health in India: a vision beckoning fulfillment? Canadian Journal of Community Mental Health 2, 131-137. 